\title{
SINGULAR INTEGRALS AND RECTIFIABILITY
}

\author{
PertTi Mattila
}

Abstract

We shall discuss singular integrals on lower dimensional subsets of $\mathbf{R}^{n}$. A survey of this topic was given in [M4]. The first part of this paper gives a quick review of some results discussed in [M4] and a survey on some newer results and open problems. In the second part we prove some results on the Riesz kernels in $\mathbf{R}^{n}$. As far I know, they have not been explicitly stated and proved, but they are very closely related to some earlier results and methods.

\section{A survey}

Not much has happened in this topic after [M4] except for the Cauchy kernel. For surveys on the development related to the Cauchy kernel, see [D2], [M5] and [V2], and for a recent fundamental contribution [T3]. One of the main goals of this survey is to emphasize the huge gap in our knowledge between the Cauchy kernel and almost any other kernel, in particular, the higher dimensional Riesz kernels. We shall mainly concentrate on general kernels and give some comments on the Cauchy kernel. Our setting consists of an $m$-dimensional Radon measure $\mu$ in $\mathbf{R}^{n}$, where $0<m<n$ is an integer, of an $m$-dimensional Calderón-Zygmund kernel $K$, and the related singular integral operator $T=T_{\mu, K}$. We shall assume that $\mu$ is Ahlfors-David (AD) regular. This means that there is $0<C<\infty$ such that

$$
r^{m} / C \leq \mu(B(x, r)) \leq C r^{m} \quad \text { for } x \in S, 0<r<\operatorname{diam}(S),
$$

where $S=\operatorname{spt} \mu$ is the support of $\mu$. Then $\mu$ is equivalent to the restriction $\mathcal{H}^{m} \mid S$ of the $m$-dimensional Hausdorff measure $\mathcal{H}^{m}$ to $S$. Thus we could as well start with a closed set $S$ and assume that $\mu=\mathcal{H}^{m} \mid S$ satisfies (1.1). In various places (1.1) could be relaxed, for example, to requirements concerning the positivity and/or finiteness of the lower

2000 Mathematics Subject Classification. 28A75, 42B20.

Key words. Singular integral, Cauchy kernel, Riesz kernel, rectifiable measure. 
and/or upper limits of $r^{-m} \mu(B(x, r))$, but we stick to (1.1) to simplify the exposition.

Also we shall not try to cover the most general class of kernels, but we shall restrict to smooth homogeneous odd kernels. Thus we assume that $K$ is of the form

$$
K(x)=|x|^{-m} \Omega(x /|x|) \quad \text { for } x \in \mathbf{R}^{n} \backslash\{0\},
$$

where $\Omega$ is a smooth function on the unit sphere $S^{n-1}$ satisfying $\Omega(-v)=$ $-\Omega(v)$.

The singular integral operator $T$ is formally given by

$$
T g(x)=\int K(x-y) g(y) d \mu y,
$$

but of course this does not usually exist for $x \in S$. There are several well-known ways to go around this to define $T$, but since all our questions reduce to the behaviour of the truncated operators $T_{\varepsilon}, \varepsilon>0$;

$$
T_{\varepsilon} g(x)=\int_{\mathbf{R}^{n} \backslash B(x, \varepsilon)} K(x-y) g(y) d \mu y,
$$

we shall not try to define $T$ itself.

Examples. 1) An AD-regular curve $\Gamma \subset \mathbf{R}^{n}$;

$$
\mathcal{H}^{1}(\Gamma \cap B(x, r)) \leq C r \quad \text { for } x \in \mathbf{R}^{n}, r>0,
$$

is an AD-regular set. The lower bound in (1.1) is automatic for curves.

2 ) The product $C=C_{1} \times C_{1} \subset \mathbf{R}^{2}$ of the $1 / 2$-dimensional symmetric Cantor set $C_{1}$ in $\mathbf{R}$ with itself is a 1-dimensional AD-regular set. That is, $C_{1}$ is constructed so that at each step the mid-half of the construction intervals is deleted.

3) The Cauchy kernel $1 / z=|z|^{-1} \Omega(z), z \in \mathbf{C}$, with $\Omega(z)=\bar{z} /|z|$, is the most important 1-dimensional kernel. Other nice 1-dimensional kernels to play with are $z^{2 k-1} /|z|^{2 k}, k=2,3,4, \ldots$.

4) Natural $m$-dimensional analogs of the Cauchy kernel are the vectorvalued Riesz kernels $|x|^{-m-1} x, x \in \mathbf{R}^{n}$.

Basic properties studied. We shall be interested in relations between three properties: rectifiability, $L^{2}$-boundedness and existence of principal values. 
Rectifiability. $\mu$ is said to be $m$-rectifiable if there are $m$-dimensional $C^{1}$ surfaces $M_{1}, M_{2}, \ldots$ such that

$$
\mu\left(\mathbf{R}^{n} \backslash \bigcup_{i=1}^{\infty} M_{i}\right)=0 .
$$

$\mu$ is purely $m$-unrectifiable if $\mu(M)=0$ for every $m$-dimensional $C^{1}$ surface $M$.

Here any reasonable definition of a surface will do, and $C^{1}$ could be replaced by Lipschitz. For the theory of rectifiability, see for example $[\mathrm{M} 3]$.

There is a quantitative notion of rectifiability of David and Semmes, called uniform rectifiability, see [DS]. We don't give the somewhat complicated general definition, but the 1-dimensional case is simple: $\mu$, or $S=\operatorname{spt} \mu$, is uniformly 1-rectifiable if there is an AD-regular curve such that $S \subset \Gamma$.

$L^{2}$-boundedness. $T=T_{k, \mu}$ is bounded in $L^{2}(\mu)$, if there is $C<\infty$ such that

$$
\int\left|T_{\varepsilon} g\right|^{2} d \mu \leq C \int|g|^{2} d \mu \quad \text { for } g \in L^{2}(\mu) \text { and for } \varepsilon>0 .
$$

This means that the operators $T_{\varepsilon}, \varepsilon>0$, are bounded in $L^{2}(\mu)$ uniformly, and it agrees with any other reasonable definition.

Existence of principal values. The principal values exist $\mu$ almost everywhere for $T=T_{\mu, k}$ if there exists

for $\mu$ almost all $x \in \mathbf{R}^{n}$.

$$
T_{\mu}(x)=\lim _{\varepsilon \rightarrow 0} \int_{\mathbf{R}^{n} \backslash B(x, \varepsilon)} K(x-y) d \mu y
$$

Three questions. The main questions we are interested in are

Q1) Does $L^{2}$-boundedness imply uniform rectifiability?

Q2) Does a.e. existence of pricipal values imply rectifiability?

Q3) Does $L^{2}$-boundedness imply a.e. existence of principal values?

The converse questions have positive answers. Recall our basic assumptions (1.1) and (1.2). Uniform rectifiability implies $L^{2}$-boundedness by the results of David and Semmes, [DS]. That rectifiability implies a.e. existence of principal values was proved by Melnikov and myself in $[\mathbf{M M}]$ for 1-dimensional sets and the Cauchy kernel. A different proof of Verdera [V1] easily generalizes to our more general setting, cf. [M3, Theorem 20.28]. 
Of course the converse of Q3 cannot quite be true, but the method of Nazarov, Treil and Volberg [NTV] gives that the a.e. existence of principal values yields the $L^{2}$-boundedness of $T$ on a subset of $S$ with large $\mu$ measure. For this it is even enough to assume that

$$
T_{\mu}^{*}(x)=\sup _{\varepsilon>0}\left|T_{\mu, \varepsilon} 1(x)\right|<\infty \quad \text { for } \mu \text { almost all } x .
$$

In fact, this finiteness of the maximal function is probably more natural assumption than the existence of principal values, but sometimes we can use the latter and not the former.

The question Q3 is amusing because one is so used to that in classical situations the answer is yes. But that is so because we can easily check it for smooth functions, which are dense in $L^{2}(\mu)$. For general AD-regular measures the existence of principal values is not automatic for any function. There are cases, discussed below, where the positive answer to Q2 is known but not to Q1. The hope is that Q3 could be used as a link.

Some answers. Very little is known about these three questions for general kernels. For the Cauchy kernel the answer to all three questions is yes, even with much less stringent conditions on $\mu$ than (1.1). The reason is the identity discovered by Melnikov in [Me]:

$$
\sum_{\sigma} \frac{1}{\left(z_{\sigma(1)}-z_{\sigma(3)} \overline{\left(z_{\sigma(2)}-z_{\sigma(3)}\right)}\right)}=c\left(z_{1}, z_{2}, z_{3}\right)^{2} ;
$$

here the sum is over all six permutations of $\{1,2,3\}$ and $c\left(z_{1}, z_{2}, z_{3}\right)$ is the so-called Menger curvature of the triple $\left\{z_{1}, z_{2}, z_{3}\right\} \subset \mathbf{C}$, which, by definition, equals $1 / R$ where $R$ is the radius of the circle passing through $z_{1}, z_{2}$ and $z_{3}\left(R=\infty\right.$ if and only if $z_{1}, z_{2}, z_{3}$ are collinear $)$. The main thing about the sum in (1.4) is not its very geometric interpretation, but that it is non-negative. This seems to be very peculiar to the Cauchy kernel and to some very closely related kernels, such as $\operatorname{Re}(1 / z)$.

The usefulness of (1.4) becomes apparent when one integrates it with respect to an AD-regular measure $\mu$. Then by Fubini's theorem and easy estimates (assuming $\mu$ has compact support)

$$
\begin{aligned}
\int\left|\int_{\mathbf{C} \backslash B(z, \varepsilon)} \frac{1}{\zeta-z} d \mu \zeta\right|^{2} d \mu z \\
=\frac{1}{6} \iiint c\left(z_{1}, z_{2}, z_{3}\right)^{2} d \mu z_{1}, d \mu z_{2}, d \mu z_{3}+O(\varepsilon) .
\end{aligned}
$$


This was used by Melnikov and Verdera in [MV] to give another proof of the boundedness of the Cauchy integral operator on Lipschitz graphs and by Melnikov, Verdera and myself in [MMV] to prove that the answer to Q1 is yes for the Cauchy kernel. That the answer to Q2 is yes was proved in [M2] (without the use of the identity (1.4)), and the yes-answer to Q3 follows from that of Q1 and the converse of Q2. So all is settled for the Cauchy kernel and AD-regular measures, but this goes much beyond them. In Q2 (1.1) can be relaxed to

$$
0<\limsup _{r \rightarrow 0} r^{-1} \mu(B(x, r))<\infty \quad \text { for } \mu \text { almost all } x \in \mathbf{C},
$$

which is essentially the same thing as to study measures $\mu=\mathcal{H}^{1} \mid E$ where $E$ is a Borel set with $\mathcal{H}^{1}(E)<\infty$. This is due to the results of Léger $[\mathbf{L}]$ and Tolsa $[\mathbf{T 2}]$. By Tolsa's results in $[\mathbf{T 1}]$ Q3 has positive answer for the Cauchy kernel and for any Radon measure $\mu$. But this is not an abstract argument, it goes through rectifiability: The $L^{2}$-boundedness implies, by a result of David, that $\mu(B(z, r)) \leq C r$ for all $z \in \mathbf{C}$, $r>0$. The set where

$$
\limsup _{r \rightarrow 0} r^{-1} \mu(B(x, r))>0
$$

has $\sigma$-finite $\mathcal{H}^{1}$ measure, and thus it is rectifiable by Léger's result (this uses the identity (1.4)). In the rest where (1.5) fails one can use that $\mu(B(x, r))$ is small for small $r$.

For other kernels much less is known. We assume again (1.1) on $\mu$. We shall discuss some partial results related to Q1 for the Riesz kernels $K_{m}$ at the end of this paper. Q2 has answer yes for $K_{m}$ by Preiss and myself, [MPr]. It has also positive answer for 1-dimensional measures $\mu$ in $\mathbf{C}$ and the kernels $z^{2 k-1} /|z|^{2 k}, k=2,3 \ldots$, by Huovinen [H1]. Both Q1 and Q2 have negative answers for example for the $\operatorname{kernel} \operatorname{Re}\left(z /|z|^{2}-z^{3} /|z|^{4}\right)$ by Huovinen's result in [H2]. By a modification of this kernel Huovinen (unpublished) has found a homogeneous kernel yielding a negative answer to Q3. Earlier David has used the Cantor set in Example 2 to construct non-homogeneous kernels giving similar counter-examples as Huovinen.

Returning to Huovinen's kernel $\operatorname{Re}\left(z /|z|^{2}-z^{3} /|z|^{4}\right)$, we observe that it vanishes both on real and imaginary axis. This is helpful in constructing the measure $\mu$ which gives the counter-example. But the situation is somewhat delicate: $\operatorname{Re}(1 / z)$ vanishes on the imaginary axis, but the same results hold for it as for the Cauchy kernel $1 / z$, since it satisfies exactly the same identity (1.4). 
Farag $[$ F2 $]$ has shown for the kernel

$$
x|x|_{p}^{-2}, \quad|x|_{p}=\left(\sum_{i=1}^{n}\left|x_{i}\right|^{p}\right)^{1 / p}, \quad x \in \mathbf{R}^{n},
$$

where $1 \leq p<\infty$, that the sum of the permutations is comparable with that of the Cauchy kernel and hence leads to the same results. On the other hand he has also shown in $[\mathbf{F 1}]$ that when $m \geq 2$ practically any reasonable modification of such sums of permutations for the Riesz kernel $K_{m}$, and any of the kernels $x|x|_{p}^{-m-1}, p \geq 1$, takes both positive and negative values and hence seems to be useless. This is the main reason for the huge gap between the results for the Cauchy kernel and for the Riesz kernels $K_{m}$ for $m \geq 2$.

\section{Partial results on the Riesz kernels}

The investigations discussed above for the Cauchy kernel are closely related to the removable sets of bounded analytic functions, see, e.g., [M3, Section 19], and [D1] for the best result so far. Similarly, the Riesz kernel $K_{n-1}$ in $\mathbf{R}^{n}$ is related to the removable singularities of Lipschitz harmonic functions, see $[\mathbf{M P}]$. Some results obtained in the study of such removable singularities in [M1] (see also [M3, Theorem 19.17]) and in $[\mathbf{M P}]$, and also the results and methods of $[\mathbf{V i}]$ indicate that the Theorems 2.1 and 2.2 below should be true. First we need the definition of tangent measures.

Let $\mu$ be a Radon measure in $\mathbf{R}^{n}$. For $a \in \mathbf{R}^{n}$ and $r>0$, let $\mu_{a, r}$ be the blow-up of $\mu$ from $B(a, r)$ to $B(0,1)$ :

$$
\mu_{a, r}(A)=\mu(r A+a), \quad A \subset \mathbf{R}^{n} .
$$

A Radon measure $\nu$ is called a tangent measure of $\mu$ at $a$, denoted $\nu \in \operatorname{Tan}(\mu, a)$, if $\nu\left(\mathbf{R}^{n}\right)>0$ and there are sequences $c_{i}>0$ and $r_{i} \downarrow 0$ such that

$$
c_{i} \mu_{a, r_{i}} \rightarrow \nu
$$

If $\mu$ satisfies (1.1), one can restrict to the sequences $\left(c r_{i}^{-m}\right)$. Knowing the measures in $\operatorname{Tan}(\mu, a)$, we know how $\mu$ looks locally. For more on these, see $[\mathbf{P}]$, where they were introduced, or [M3, Section 14].

Theorem 2.1. Let $\mu$ be an AD-regular m-dimensional Radon measure in $\mathbf{R}^{n}$. If $T_{\mu, K_{m}}$ is bounded in $L^{2}(\mu)$, then for $\mu$ almost all $a \in \mathbf{R}^{n}$, $\operatorname{Tan}(\mu, a)$ contains some measure $\nu$ of the form $\nu=\mathcal{H}^{m} \mid V$, where $V$ is an affine m-plane. 
In other words, this means that around $\mu$ almost all points $a$ at some (but not necessarily all) arbitrarily small scales $S=\operatorname{spt} \mu$ is very well approximable by $m$-planes. In particular, $S$ cannot be a kind of $m$-dimensional analogue of the Cantor set $C$ in Example 2.

A duality method and some standard estimates, cf. e.g. [M3, Section 20], show that there is $g \in L^{\infty}(\mu), g \neq 0, g \geq 0$, such that the maximal function (recall (1.3)) $T_{\mu, K_{m}}^{*} g$ is bounded. Thus Theorem 3.1 follows from

Theorem 2.2. Let $\mu$ be a Radon measure in $\mathbf{R}^{n}$ such that for $\mu$ almost all $x \in \mathbf{R}^{n}$,

$$
0<\liminf _{r \rightarrow 0} r^{-m} \mu(B(x, r)) \leq \limsup _{r \rightarrow 0} r^{-m} \mu(B(x, r))<\infty .
$$

If for $\mu$ almost all $x \in \mathbf{R}^{n}$,

$$
\sup _{0<\varepsilon<1}\left|\int_{B(x, 1) \backslash B(x, \varepsilon)}\right| x-\left.y\right|^{-m-1}(x-y) d \mu y \mid<\infty,
$$

then for $\mu$ almost $a \in \mathbf{R}^{n}$ there is $\nu \in \operatorname{Tan}(\mu, a)$ of the form $\nu=c \mathcal{H}^{m} \mid V$, where $c>0$ and $V$ is an m-plane.

Proof: It is not hard to see, cf. Lemmas 14.7 and 19.15 in [M3], that for $\mu$ almost all $a \in \mathbf{R}^{n}$, every $\nu \in \operatorname{Tan}(\mu, a)$ is an $m$-dimensional AD-regular measure such that

$$
\sup _{0<r<R<\infty}\left|\int_{B(x, R) \backslash B(x, r)}\right| x-\left.y\right|^{-m-1}(x-y) d \nu y \mid<\infty
$$

for $x \in \operatorname{spt} \nu$.

Since $m<n$ and $\nu$ is $m$-regular, spt $\nu \neq \mathbf{R}^{n}$. Thus we can find $b, c \in \mathbf{R}^{n}$ and $r>0$ such that $(\operatorname{Int} B(b, r)) \cap \operatorname{spt} \nu=\emptyset$ and $c \in(\partial B(b, r)) \cap \operatorname{spt} \nu$. Let $\lambda \in \operatorname{Tan}(\nu, b)$. Then $0 \in \operatorname{spt} \lambda$ and $\operatorname{spt} \lambda \subset\left\{x \in \mathbf{R}^{n}: x \cdot(c-b) \geq\right.$ $0\}$, as one easily verifies. Also $\lambda$ satisfies (2.1) in place of $\nu$. This implies that $\operatorname{spt} \lambda$ is contained in the hyperplane $\{x: x \cdot(c-b)=$ $0\}$. By [M3, Theorem 14.16], $\lambda \in \operatorname{Tan}(\mu, a)$ for $\mu$ almost all starting points $a$. Continuing this dimension deduction argument we arrive at an $m$-dimensional $\mathrm{AD}$-regular $\sigma \in \operatorname{Tan}(\mu, a)$ whose support is contained in an $m$-plane $V$. By the AD-regularity, $\sigma$ is absolutely continuous with respect to $\mathcal{H}^{m}\left|V ; \sigma=h \mathcal{H}^{m}\right| V, h \in L^{\infty}\left(\mathcal{H}^{m} \mid V\right)$. Taking one more tangent measure at a point $z$ where $h(z) \neq 0$ and $h$ is approximately continuous, we find the required tangent measure of $\mu$ at $a$. 


\section{References}

[D1] G. DAvID, Unrectifiable 1-sets have vanishing analytic capacity, Rev. Mat. Iberoamericana 14(2) (1998), 369-479.

[D2] G. DAvid, Analytic capacity, Calderón-Zygmund operators, and rectifiability, Publ. Mat. 43(1) (1999), 3-25.

[DS] G. David AND S. SEmmes, "Analysis of and on uniformly rectifiable sets", Mathematical Surveys and Monographs 38, American Mathematical Society, Providence, RI, 1993.

[F1] H. M. FARAG, The Riesz kernels do not give rise to higherdimensional analogues of the Menger-Melnikov curvature, Publ. Mat. 43(1) (1999), 251-260.

[F2] H. M. FARAG, Curvatures of the Melnikov type, Hausdorff dimension, rectifiability, and singular integrals on $\mathbf{R}^{n}$, Pacific $J$. Math. 196(2) (2000), 317-339.

[H1] P. Huovinen, Singular integrals and rectifiability of measures in the plane, Ann. Acad. Sci. Fenn. Math. Diss. 109 (1997), $63 \mathrm{pp}$.

[H2] P. Huovinen, A nicely behaved singular integral on a purely unrectifiable set, Proc. Amer. Math. Soc. 129(11) (2001), $3345-3351$.

[L] J. C. LÉGER, Menger curvature and rectifiability, Ann. of Math. (2) 149(3) (1999), 831-869.

[M1] P. Mattila, A class of sets with positive length and zero analytic capacity, Ann. Acad. Sci. Fenn. Ser. A I Math. 10 (1985), 387-395.

[M2] P. MAtTila, Cauchy singular integrals and rectifiability in measures of the plane, Adv. Math. 115(1) (1995), 1-34.

[M3] P. MATTILA, "Geometry of sets and measures in Euclidean spaces. Fractals and rectifiability", Cambridge Studies in Advanced Mathematics 44, Cambridge University Press, Cambridge, 1995.

[M4] P. MatTiLA, Singular integrals, analytic capacity and rectifiability, in: "Proceedings of the conference dedicated to Professor Miguel de Guzmán" (El Escorial, 1996), J. Fourier Anal. Appl. 3, Special Issue (1997), 797-812.

[M5] P. Mattila, Rectifiability, analytic capacity, and singular integrals, in: "Proceedings of the International Congress of Mathematicians", Vol. II (Berlin, 1998), Doc. Math. Extra Vol. II (1998), 657-664 (electronic). 
[MM] P. Mattila and M. S. Melnikov, Existence and weak-type inequalities for Cauchy integrals of general measures on rectifiable curves and sets, Proc. Amer. Math. Soc. 120(1) (1994), $143-149$.

[MMV] P. Mattila, M. S. Melnikov and J. Verdera, The Cauchy integral, analytic capacity, and uniform rectifiability, Ann. of Math. (2) 144(1) (1996), 127-136.

[MP] P. Mattila and P. V. Paramonov, On geometric properties of harmonic Lip ${ }_{1}$-capacity, Pacific J. Math. 171(2) (1995), 469-491.

[MPr] P. Mattila and D. Preiss, Rectifiable measures in $\mathbf{R}^{n}$ and existence of principal values for singular integrals, J. London Math. Soc. (2) 52(3) (1995), 482-496.

[Me] M. S. Melnikov, Analytic capacity: a discrete approach and the curvature of measure, (Russian), Mat. Sb. 186(6) (1995), 57-76; translation in Sb. Math. 186(6) (1995), 827-846.

[MV] M. S. Melnikov and J. Verdera, A geometric proof of the $L^{2}$ boundedness of the Cauchy integral on Lipschitz graphs, Internat. Math. Res. Notices 1995(7) (1995), 325-331.

[NTV] F. Nazarov, S. Treil and A. Volberg, Pulling ourselves up by the hair, Preprint.

[P] D. Preiss, Geometry of measures in $\mathbf{R}^{n}$ : distribution, rectifiability, and densities, Ann. of Math. (2) 125(3) (1987), 537-643.

[T1] X. TOLSA, Cotlar's inequality without the doubling condition and existence of principal values for the Cauchy integral of measures, J. Reine angew. Math. 502 (1998), 199-235.

[T2] X. Tolsa, Principal values for the Cauchy integral and rectifiability, Proc. Amer. Math. Soc. 128(7) (2000), 2111-2119.

[T3] X. Tolsa, Painlevé's problem and the semiadditivity of the analytic capacity, Preprint.

[V1] J. VerderA, A weak type inequality for Cauchy transforms of finite measures, Publ. Mat. 36(2B) (1992), 1029-1034.

[V2] J. Verdera, $L^{2}$ boundedness of the Cauchy integral and Menger curvature, in: "Harmonic analysis and boundary value problems" (Fayetteville, AR, 2000), Contemp. Math. 277, Amer. Math. Soc., Providence, RI, 2001, pp. 139-158. 
[Vi] M. Vintilä, The boundedness of Riesz $s$-transforms of measures in $\mathbf{R}^{n}$, Proc. Amer. Math. Soc. 124(12) (1996), 3797-380.

Department of Mathematics and Statistics

University of Jyväskylä

P. O. Box 35 (MaD)

FIN-40351 Jyväskylä

Finland

E-mail address: pmattila@maths.jyu.fi 